\title{
The Emergence Characteristics of Driver's Intentions Influenced by Different Emotions
}

\author{
Xiaoyuan Wang ${ }^{1,2, *}$, , Yongqing Guo ${ }^{2,3}$, Chenglin Bai ${ }^{4}$, Quan Yuan ${ }^{5}$, Shanliang Liu ${ }^{1}$ and Xuegang (Jeff) Ban ${ }^{6}$ \\ 1 College of Electromechanical Engineering, Qingdao University of Science \& Technology, \\ Qingdao 266000, China; liushanliang@mails.qust.edu.cn \\ 2 Joint Laboratory for Internet of Vehicles, Ministry of Education-China Mobile Communications Corporation, \\ Tsinghua University, Beijing 100084, China; yongqing.guo@sdut.edu.cn \\ 3 School of Transportation and Vehicle Engineering, Shandong University of Technology, Zibo 255000, China \\ 4 School of Physics Science and Communication Engineering, Liaocheng University, Liaocheng 252000, China; \\ baichenglin@lcu.edu.cn \\ 5 State Key Laboratory of Automotive Safety and Energy, School of Vehicle and Mobility, Tsinghua University, \\ Beijing 100084, China; yuanq@tsinghua.edu.cn \\ 6 Department of Civil and Environmental Engineering, University of Washington, Seattle, WA 98195, USA; \\ banx@uw.edu \\ * Correspondence: wangxiaoyuan@qust.edu.cn
}

check for

updates

Citation: Wang, X.; Guo, Y.; Bai, C.; Yuan, Q.; Liu, S.; Ban, X. The Emergence Characteristics of Driver's Intentions Influenced by Different Emotions. Sustainability 2021, 13, 13292. https://doi.org/10.3390/ su132313292

Academic Editors: Xiaobei Jiang, Haixiang Lin, Fei Yan and Qian Cheng

Received: 9 October 2021

Accepted: 26 November 2021

Published: 1 December 2021

Publisher's Note: MDPI stays neutral with regard to jurisdictional claims in published maps and institutional affiliations.

Copyright: (c) 2021 by the authors. Licensee MDPI, Basel, Switzerland. This article is an open access article distributed under the terms and conditions of the Creative Commons Attribution (CC BY) license (https:/ / creativecommons.org/licenses/by/ $4.0 /)$.

\begin{abstract}
Drivers' behavioral intentions can affect traffic safety, vehicle energy use, and gas emission Drivers' emotions play an important role in intention generation and decision making. Determining the emergence characteristics of driver intentions influenced by different emotions is essential for driver intention recognition. This study focuses on developing a driver's intention emergence model with the involvement of driving emotion on two-lane urban roads. Driver emotions were generated using various ways, including visual stimuli (video and picture), material incentives, and spiritual rewards. Real and virtual driving experiments were conducted to collect the multi-source dynamic data of human-vehicle-environment. The driver intention emergence model was constructed based on an artificial neural network, to identify the influences of drivers' emotions on intention, as well as the evolution characteristics of drivers' intentions in different emotions. The results show that the proposed model can make accurate predictions on driver intention emergence. The findings of this study can be used to improve drivers' behavior, in order to create more efficient and safe driving. It can also provide a theoretical foundation for the development of an active safety system for vehicles and an intelligent driving command system.
\end{abstract}

Keywords: driver's emotion; driving intention; emergence characteristics; artificial neural network; active safety system

\section{Introduction}

Drivers' behavioral intentions could have a significant effect on traffic safety, fuel use, and gas emission. Drivers' intentions are closely related to their emotions while operating a vehicle. For example, when complimented by friends or passengers in the car, a young driver might feel complacent, leading to dramatic accelerating and frequent lane changing. These driving behaviors increase crash risk, fuel consumption, and exhaust emission. Driving emotion can be considered as a reaction to the surrounding environment, which can affect a driver's cognition and judgment, and also a driver's intention [1]. Therefore, it is necessary to reveal the mechanism of effect of emotion on intention to improve their behaviors, in order to encourage more efficient and safe driving patterns.

In recent years, research on emotion-intention interaction has mainly focused on sports, shopping consumption, environmental protection, moral education, and so on. For example, Mohiyeddini et al. [2] evaluated sport intention identification models, and found that the intention recognition performance can be enhanced by considering emotional 
factors. Chen et al. [3] used the structural equation model to identify the effects of nostalgia on consumption intention in restaurants. The results showed that the consumption intention is directly or indirectly influenced by nostalgia, and the influence varies from person to person. Ladhari et al. [4] recognized customer purchase intentions according to their perceptions of service quality and emotional responses to receiving services. The results showed that service quality and environment have a strong influence on customers' emotional satisfaction. Higher emotional satisfaction results in greater purchase probability. Harth et al. [5] examined the influence of three emotions of guilt, pride, and anger on the environmental intentions. The result revealed that guilt makes people more prone to produce the intention to reconstruct the environment, anger makes people more prone to produce the intention to punish the environmental destroyer, and pride makes people more prone to produce the intention to protect the environment. Yang et al. [6] analyzed the relationship among visitors' behavior intention at festivals, consumption emotion and perceived value, as well as the moderating effect of the festival environment on the influence of tourists' consumption emotion on behavioral intention. Chen et al. [7] used the clinical interview method to explore the influences of various situations on moral judgment and emotion attribution in children.

Only a few works thus far demonstrate approaches in driver intention prediction. Lethaus et al. [8] compared several supervised learning algorithms to infer driver intention according to eye movement data. Helman et al. [9] discussed the changes in self-reported behavioral intentions between the pre-license learning stage and the post-license driving period. Waddell et al. [10] used the theory of planned behavior and extended descriptive norms to investigate the psychosocial influence on driver's intentions. Auzoult et al. [11] used a questionnaire to examine drivers' behavioral intentions related to speeding and drinking. Wang et al. [12] proposed a conflict point method to analyze the impact of bicycles on driving behavior in a mixed traffic environment.

In conclusion, the effects of human emotion on behavior and intention have been widely explored in different research fields. However, research on the influence mechanism of a driver's emotion on intention still remains largely unexplored in the field of safety and efficiency of urban traffic [11-14]. In this study, we developed a driver intention evolution model to identify the emergence characteristics of drivers' intentions in different types of emotions. The real and virtual driving experiments were carried out under the conditions of different driver emotions to collect the multi-source data on the humanvehicle-environment dynamic.

\section{Materials and Methods}

\subsection{Analysis of Vehicle Group Relationship}

The vehicle group is composed of dynamic traffic entities, which can construct specific traffic scenes for driving experiments. To explore the impacts of a driver's emotion states on driving intention in diverse traffic situations, various scenarios of vehicle group were defined, shown in Figure 1. When the target vehicle was operated by the subject in the right lane, the interest-sensitive area was divided into front, left-front, left-rear, and rear according to the horizontal line of the target vehicle's front bumper and the line separating the two lanes in the same direction. The interest-sensitive area is described as the area with the greatest impact on vehicle safety and driver attention. When the target vehicle was operated in the left lane, the interest-sensitive area was divided into front, right-front, right-rear, and rear sides [15-17]. 


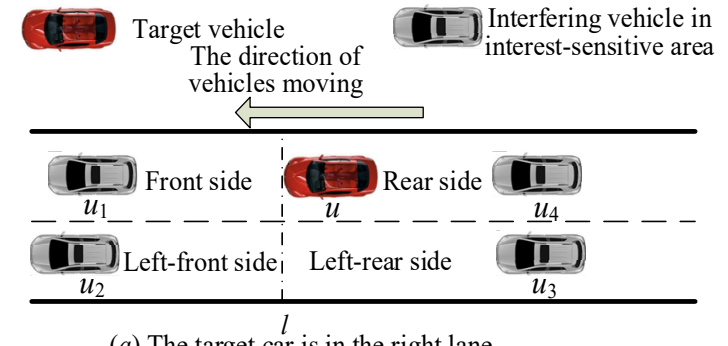

(a) The target car is in the right lane

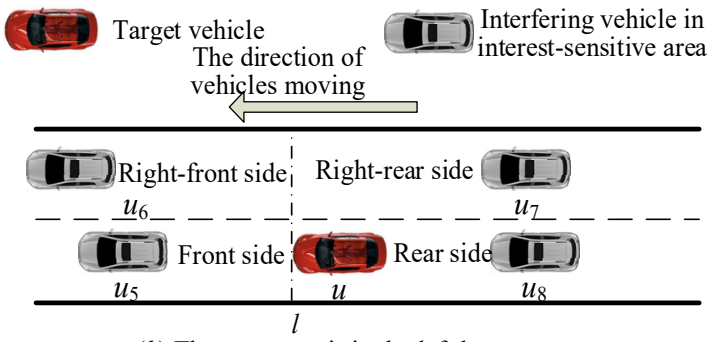

(b) The target car is in the left lane

Figure 1. Vehicle groups in a two-lane environment.

The concept of "force" was borrowed to describe the effect of the magnitude and direction of the neighboring vehicles on the target vehicle $[15,16]$. Six determinants were used for analysis in the interest-sensitive area, namely gender, driving experience, determination, propensity, and relative distances and relative speeds between target vehicle and surrounding vehicles [16-19]. In addition, based on a group of "forces" on vehicles, the fuzzy logic method was used to abstractly represent the vehicle group relationship. If a vehicle pushed the target vehicle to leave the current lane, it could be considered that the vehicle exerted a "repulsive force" on the target vehicle. The opposite situation was considered as an "attraction force". Ultimately, eight vehicle group situations were obtained, shown in Figure 2. The eight vehicle group situations are denoted as T1-T8, and the attraction and repulsion forces were denoted by "+" and "-", respectively [16].

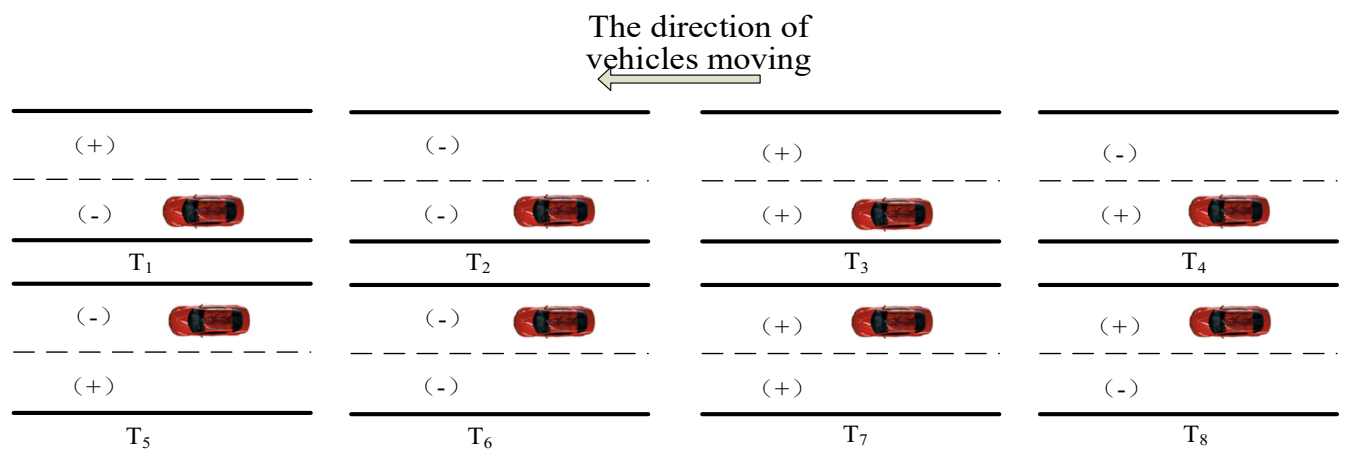

Figure 2. Vehicle group relationship when the target vehicle locates in the left and right lanes.

\subsection{Driver Intention Analysis Model in Different Emotions-Learning Algorithm of Three-Layer Feedforward Neural Networks}

Neural networks are multi-layer networks of neurons [20]. For a basic neuron model, the input is $x_{i}(i=1,2, \ldots, n)$, and the output is $y$. Then, $y=f\left(\sum_{i=1}^{n} \omega_{i} x_{i}-\theta_{i}\right)$, where $\omega_{i}$ represents the connection weight of the current neuron and the previous neuron, and $\theta_{i}$ represents the threshold [21].

Figure 3 shows the structural diagram of three-layer feedforward neural network [21]. This network is composed of one input layer, one hidden layer, and one output layer. $w_{i j}$ stands for the connection weight of input and hide layers, and $w_{j k}$ stands for the connection weight of hide and output layers. The sigmoid function is used to perform a transform in neural networks, and its expression is $(u)=\frac{1}{1+e^{-u}}$. In this study, the backpropagation algorithm (B-P algorithm) is used for the learning of the three-layer forward neural network model. A set of training samples is given, each of which contains inputs and expected outputs. When all the actual outputs are the same as the expected outputs, the training ends. Otherwise, by correcting the weight value, the expected outputs match with the actual outputs [21]. 


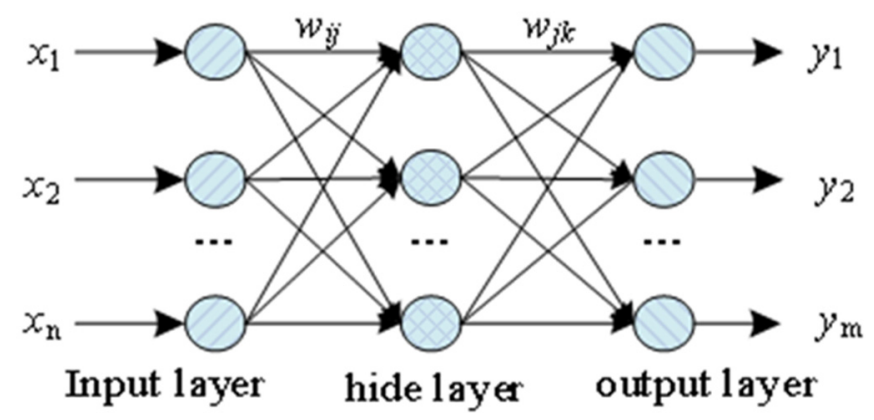

Figure 3. Structural diagram of three-layer feedforward neural network.

\subsection{Application of Forward Neural Network on Driver's Intention}

The neural network model has been considered as an efficient tool for forecasting driver behavior and travel demand [22,23]. In this study, driver emotion and vehicle group are used as input parameters of the neural network, and the probability of driver intention with emotion is taken as output. According to the revised PAD Emotional Scale model developed by the Chinese Academy of Sciences, driver emotion is expressed in 3 dimensions: pleasure (P), arousal (A), and dominance (D) [24]. The vehicle group relationship is expressed by the "forces" exerted on the target vehicle from the surrounding vehicles in the interest-sensitive area of the two lanes. The weight values are adjusted by the three-layer feedforward neural networks, and the three-layer forward neural network model is trained based on the B-P algorithm. The expected output of driver intention probability in the learning process is obtained by statistical analysis of the experimental data, and the learning process is shown in Figure 4.

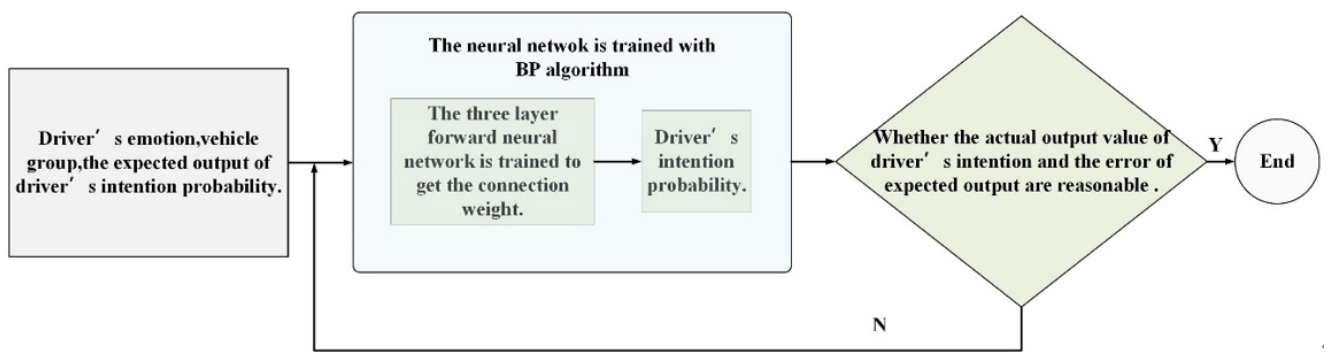

Figure 4. Neural network model for analyzing driver intention emergence characteristics.

\section{Experimental Design}

The driving experiments in this study include real and virtual driving. The real driving experiments can collect more reliable data on a driver's behavior and emotion in real traffic situations. However, performing an experiment in a real traffic environment is expensive, time-consuming, and less safe. The virtual driving experiment was used as a substitute for real vehicle experiments, due to its low cost, reproducibility, and safety. Road Builder and UC-win/Road software were applied in the high-fidelity driving simulator to create a realistic 3D traffic environment platform, based on the road attributes, traffic volume, and other parameters of the field driving experiments. The selected driving experiment route was a straight line with a total length of $4700 \mathrm{~m}$, shown in Figure 5.

Seven kinds of driver intentions were analyzed in this study: going straight at a constant speed (I1), going straight with acceleration (I2), going straight with deceleration (I3), lane changing at a constant speed (I4), lane changing with acceleration (I5), lane changing with deceleration (I6), and stopping (I7). Eight types of common emotions were included: fear, helplessness, relief, pleasure, surprise, anxiety, contempt, and anger. It should be noted that driver intentions were selected by analyzing the vehicle driving status in a two-lane environment. Driver emotions were selected based on the emotion classification proposed by Johnston [24]. This classification approach for emotional responses is widely accepted. Johnston suggested that we have eight basic emotions, and all other emotions are 
made up by combining these basic emotions. Drivers mostly experience these fundamental emotions in driving environments. The experimental design is shown in Table 1.

Table 1. Experimental design.

\begin{tabular}{|c|c|c|}
\hline Items & Real Driving Experiment & Virtual Driving Experiment \\
\hline Experimental materials & \multicolumn{2}{|c|}{$\begin{array}{l}\text { Psychometric questionnaire of driver's propensity [15,16], psychometric questionnaire of } \\
\text { willpower [25], International Affective Picture System (IAPS), Chinese Affective Picture } \\
\text { System (CAPS) }\end{array}$} \\
\hline Experimental equipment & $\begin{array}{l}\text { Human factor engineering experiment system, GPS } \\
\text { high-precision positioning system, 32-Wire LiDAR, } \\
\text { SG299GPS Non-contact multi-function speedometer, } \\
\text { Video-capturing system, etc. }\end{array}$ & $\begin{array}{l}\text { Interactive Parallel Driving Virtual } \\
\text { Experiment Platform, Human factor } \\
\text { engineering experiment system, } \\
\text { Video-capturing system. }\end{array}$ \\
\hline $\begin{array}{l}\text { Experimental } \\
\text { condition }\end{array}$ & $\begin{array}{l}\text { Off-peak periods of sunny days and appropriate } \\
\text { road conditions }\end{array}$ & Virtual driving laboratory \\
\hline Route & $\begin{array}{l}\text { Zhangzhou road between Jiangmeng road and } \\
\text { Shanshen line in Zhangdian District of Zibo city, } \\
\text { Shandong province (shown in Figure 5). }\end{array}$ & $\begin{array}{l}\text { Road scene edited by Interactive Parallel } \\
\text { Driving Virtual Experiment Platform } \\
\text { according to the real driving experiment } \\
\text { route. }\end{array}$ \\
\hline Subjects & \multicolumn{2}{|c|}{$\begin{array}{l}\text { Fifty-four drivers, } 27 \text { males and } 27 \text { females, were selected to take part in the experiments. Their ages } \\
\text { ranged between } 18 \text { and } 70 \text { years. The average age was } 33.5 \text { years old. The average driving mileage } \\
\text { was } 12,000 \mathrm{~km} \text {. }\end{array}$} \\
\hline & \multicolumn{2}{|c|}{$\begin{array}{l}\text { Driver's emotions were stimulated with pictures, video, audio, and other materials in IAPS and } \\
\text { CAPS (Some of the emotional materials were shown in Figure 6). Different method combinations } \\
\text { were used to evoke different emotions in the experimental scenes [26]. }\end{array}$} \\
\hline Emotion induction & $\begin{array}{l}\text { Angry emotion was stimulated by using abusive and } \\
\text { offensive words. Surprise emotion was stimulated } \\
\text { by telling the world Trolltech event, releasing the } \\
\text { scent of peppermint, and watching the images of } \\
\text { surprised faces. Fear emotion was stimulated by } \\
\text { telling horror stories or watching videos of terrorist } \\
\text { traffic accidents, and recalling scary experiences. } \\
\text { Helplessness was induced by watching film clips of } \\
\text { desperation and helplessness, and writing about } \\
\text { cases from his/her past when he/she experienced } \\
\text { stress. Anxiety and contempt emotions were } \\
\text { stimulated by speech induction, psychological hints, } \\
\text { etc. Relief emotion was stimulated by sharing happy } \\
\text { experiences. Pleasure emotion was stimulated by } \\
\text { talking, giving some gifts, smiling, singing, or } \\
\text { reading beautiful poems. }\end{array}$ & $\begin{array}{l}\text { Besides the above-mentioned approaches, a } \\
\text { few traffic scenes were designed for the } \\
\text { virtual driving experiment. Fear, anxiety, and } \\
\text { anger were stimulated with traffic scenes, } \\
\text { such as a traffic accident, honking horn } \\
\text { suddenly, waiting for a long time in } \\
\text { intersections, or crowded vehicle roads, in on } \\
\text { the Interactive Parallel Driving Virtual } \\
\text { Experiment Platform. Surprise emotion was } \\
\text { stimulated through changing the weather } \\
\text { and environment in the virtual scenes. Relief } \\
\text { and pleasure emotions were stimulated } \\
\text { through setting a comfortable traffic } \\
\text { environment (such as Green Wave). } \\
\text { Contempt emotion was stimulated using race } \\
\text { driving scenes. }\end{array}$ \\
\hline
\end{tabular}

Keeping and increasing emotional level

Assessing the level of the induced emotions
The driver's emotional level was kept and increased through multiple ways of listening to the music, communicating, recalling memories, etc., during driving.

Before and after the driving experiment, each driver was asked to evaluate his/her own emotional levels. A driver's physiological signals of electrocardiography (ECG), electrodermal activity (EDA), and skin temperature (SKT) were collected using PsyLAB during driving. The physiological signals were used as auxiliary to assess emotional levels, through the method developed by Platt [27]. 


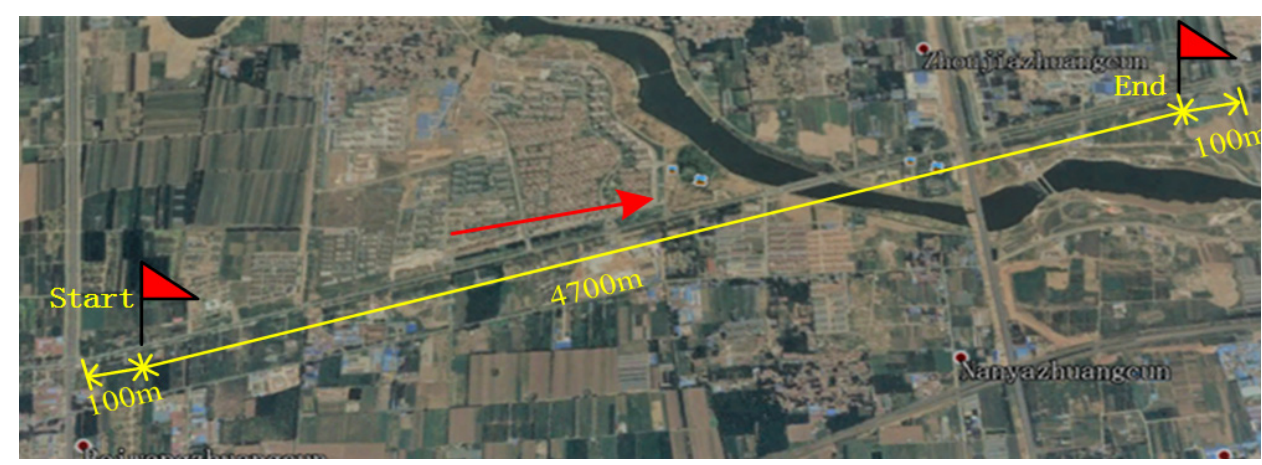

Figure 5. Real driving experiment route.

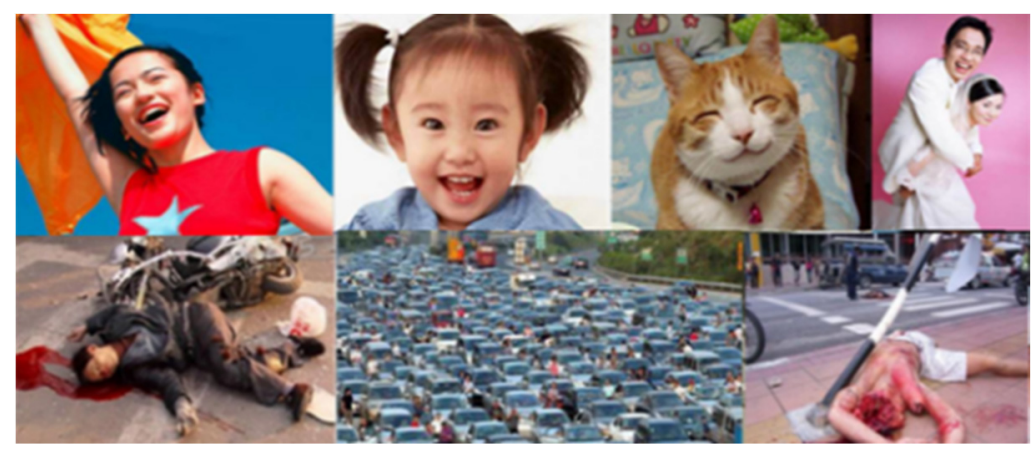

Figure 6. Parts of driver emotion visual stimulus material. (Source: International Affective Picture System \& Chinese Affective Picture System).

Participants' personal information and driving-related information were collected before starting the experiment, including age, gender, propensity, vehicle mileage traveled, and driving style. Participants were told about the completed experimental procedure, and were asked to learn how to manipulate the experimental vehicle. The International Affective Picture System (IAPS) and the Chinese Affective Picture System (CAPS) were used as emotional induction materials (A few emotional pictures were shown in Figure 6). Before running each driving experiment, the subject's emotion was induced to a certain level of arousal in the lab. Only one subject was involved at a time, under the supervision of two researchers. In the first few minutes, the subject was asked to listen relaxing music and watch images of non-threatening animals, to place him/her in a neutral mood. Then, the subject's emotions were stimulated using various approaches, including watching static images or videos, listening to certain music, autobiographical recall, and situational procedures. When the subject's emotion was induced to a certain arousal level or above, the driving experiment began. In the process of the driving experiment, the relevant music was played or an odor was released to maintain and raise the level of emotional arousal (shown in Table 1). During the experiment, the driver's facial expression and action, road conditions, driving speed, and pedal strength were recorded in real time with the video monitoring system, speedometer, and pedal dynamics instrument. When the driving experiments were completed, participants were asked to watch the recorded video, and describe their emotional states in the experiments. The equipment used in the real driving experiment and the virtual drivng experiment are illustrated in Figures 7 and 8. 


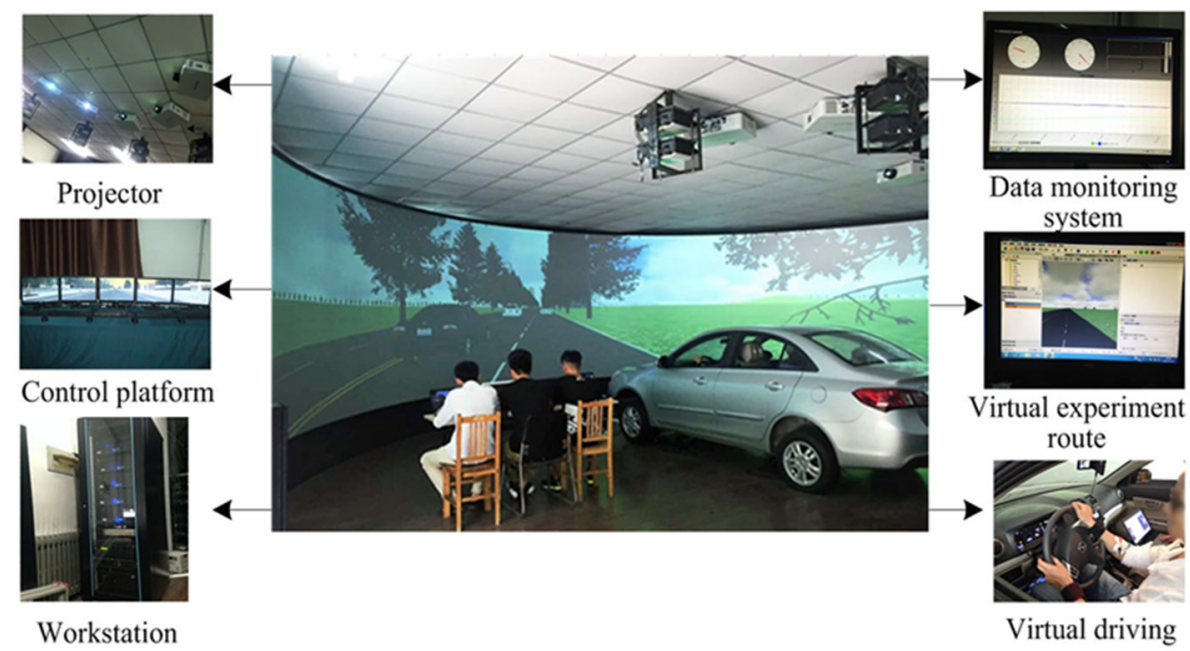

Figure 7. Virtual driving experiment (from Wang et al. [16]).

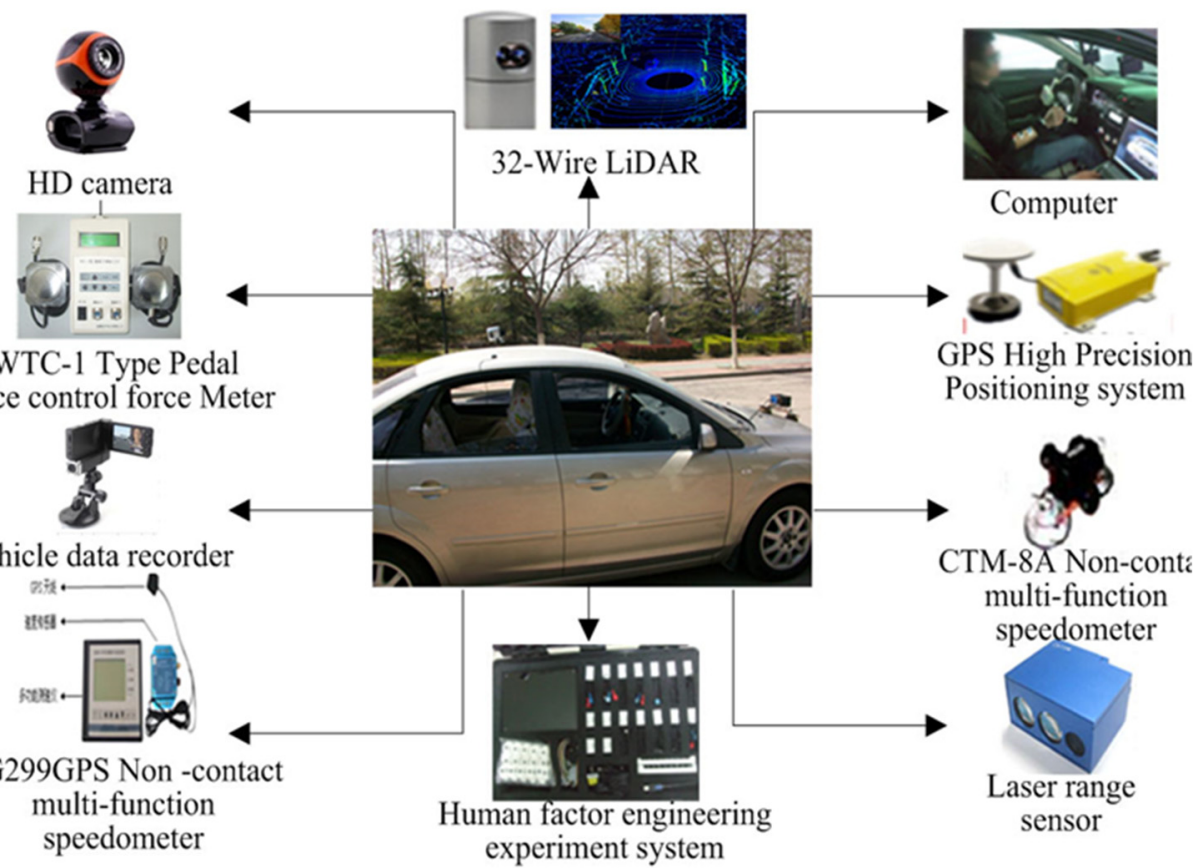

Figure 8. Real driving experiment (from Wang et al. [16]).

\section{Results}

In total, 1000 groups of effective data for each driving intention were selected to train the neural network. The error threshold of neural network learning was set as $10^{-10}$, and the number of learning cycles was 1000. In the process of model learning, the sigmoid function was used to transform a neuron into an output, and there was one node in the output layer. The error curves of driver intentions in the learning process are shown in Figure 9. The horizontal axis shows the number of learning cycles, and the vertical axis shows the error value. It was observed that after running approximately 250 learning cycles, the error of each driving intention fell below the threshold value $\left(10^{-10}\right)$.

Apart from the data for training the neural network, the remaining were used as testing samples. A total of 7000 groups of effective data were used for the testing samples to assess the effectiveness of the model (1000 sets for each driving intention). The model outputs were compared to the experimental results for each driving intention. As shown in Figure 10, the model can achieve over $75 \%$ accuracy. 

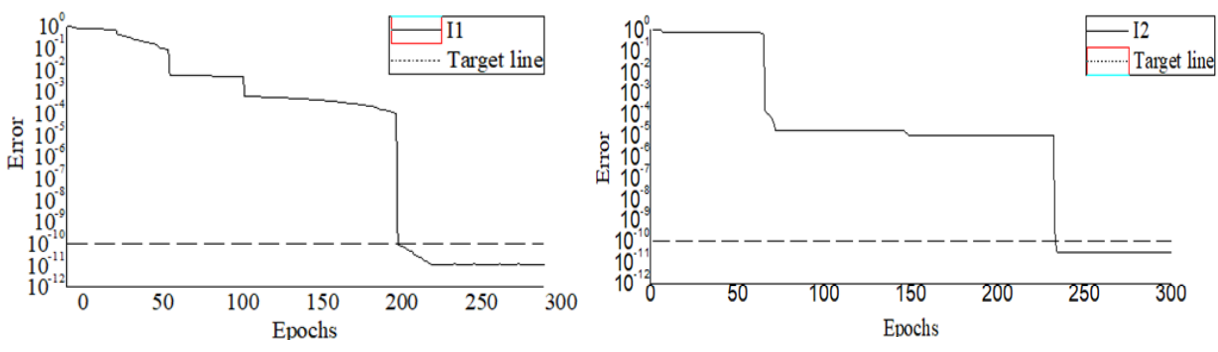

I1

I2
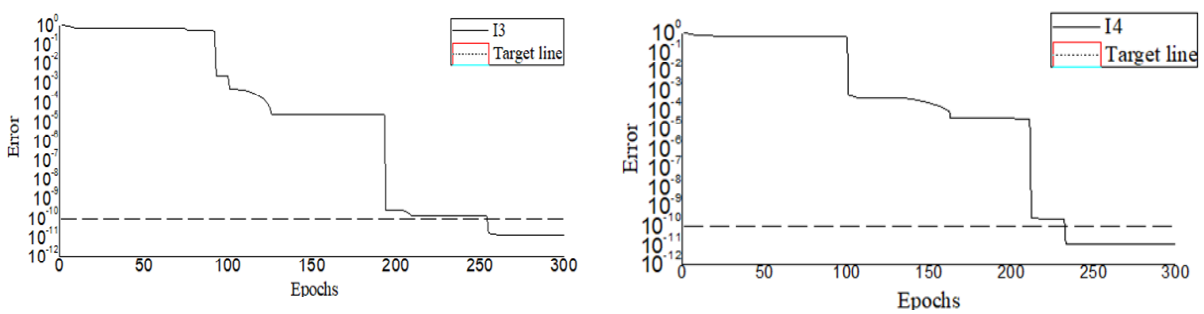

I3

I4
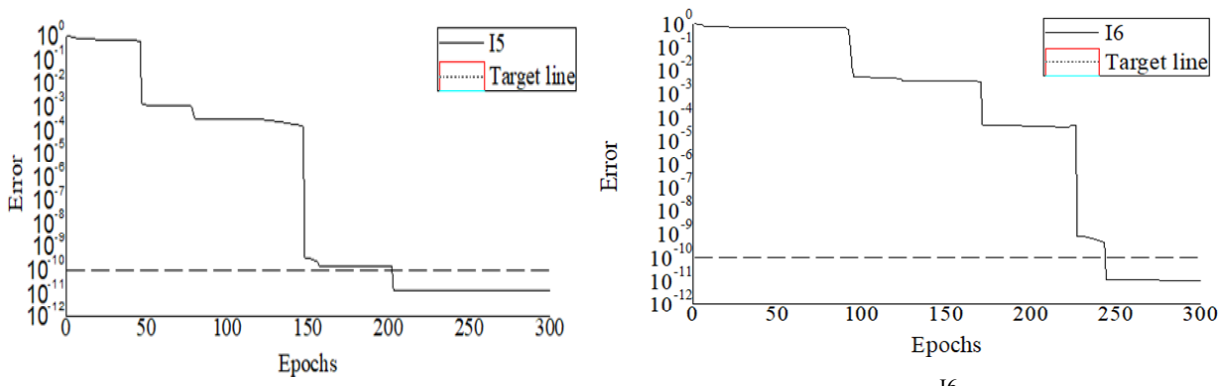

I5

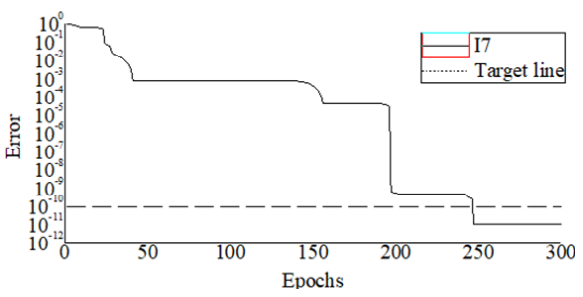

I7

Figure 9. Error curves of driver intentions in the learning process.
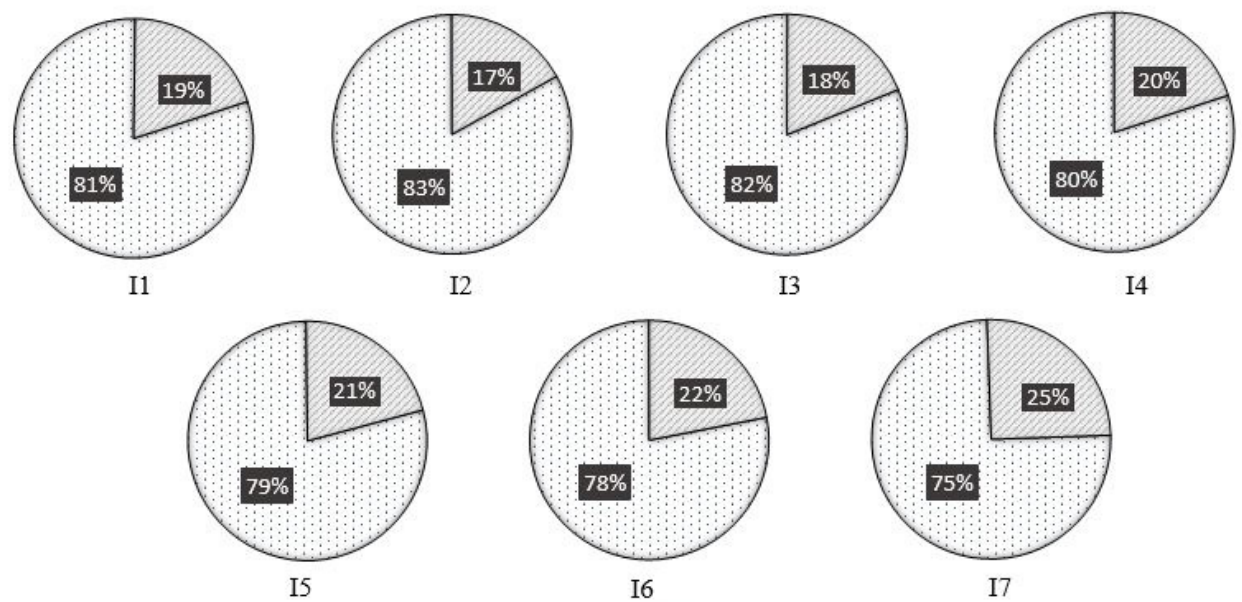

Consistent

Inconsistent

Figure 10. Comparison of algorithm results and experimental results. 
The error curves of neural network in the testing process are shown in Figure 11. The ordinate shows the test error value, and the abscissa shows the number of cycles. When the actual error reaches $1.14781 \times 10^{-11}$ (below the threshold value $10^{-10}$ ), the test is terminated.

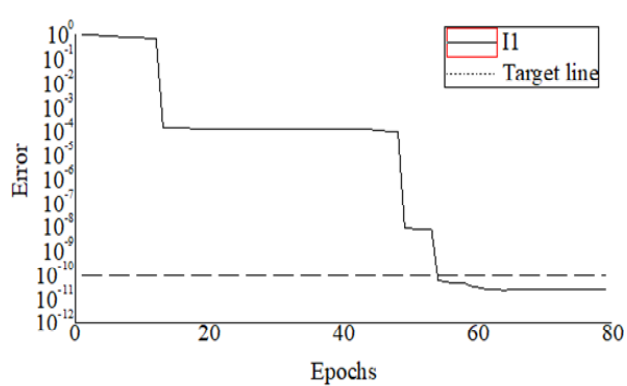

I1

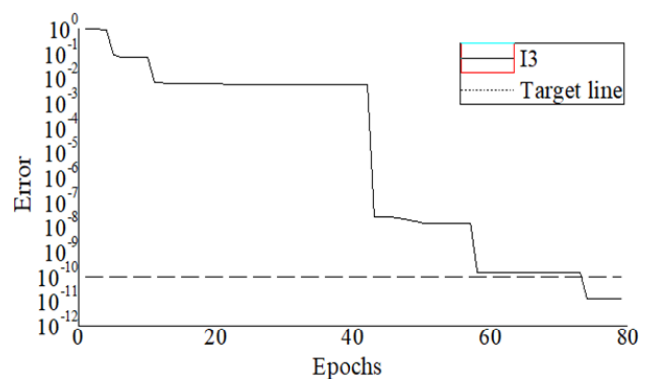

I3

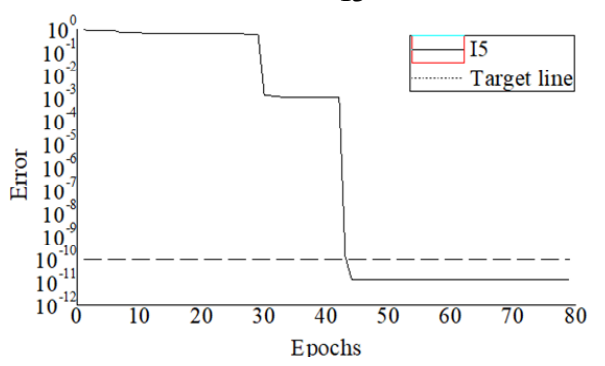

I5

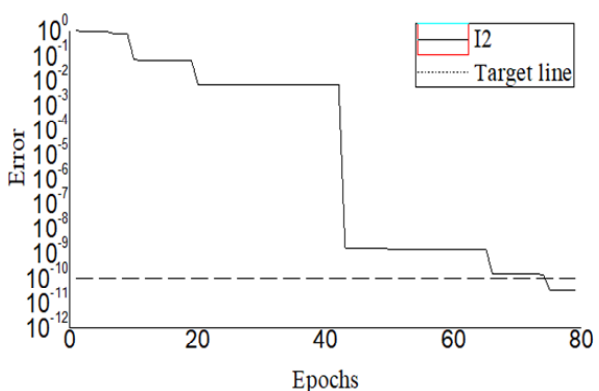

I2

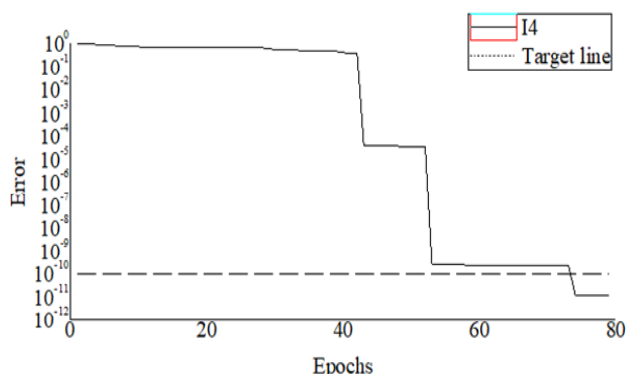

I4

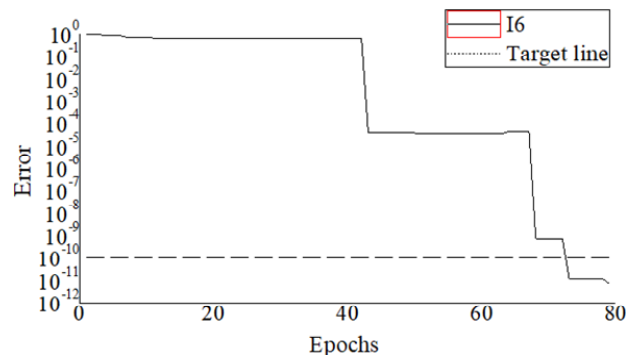

I6

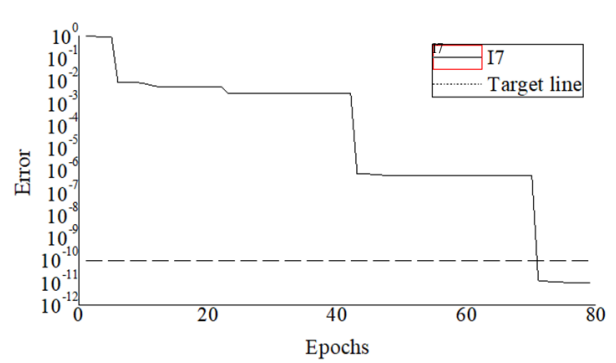

I7

Figure 11. Error curves of driver intentions in the testing process.

Comparing Figure 9, Figure 11, it can be seen that at least 200 cycles are required to achieve the desired accuracy in the learning process, while less than 80 cycles are required during the testing process. This is because the training process makes the structure of the neural network more stable, so the test error can quickly fall below the threshold. To sum up, the developed model for predicting a driver's intention emergence characteristics can achieve accurate results.

\section{Discussion}

There are significant differences in the characteristics of driver intention emergence under different emotions. In this paper, vehicle groups T1, T3, and T5 were used as examples to illustrate the probabilities of a driver's intention in different emotional states, 
shown in Figures 12-14. The numbers of 1, 2, 3, 4, 5, 6, 7, and 8 on the $x$-axis represent the emotions of fear, helplessness, relief, pleasure, surprise, anxiety, contempt, and anger, respectively. The $y$-axis represents the probability of driver intentions.

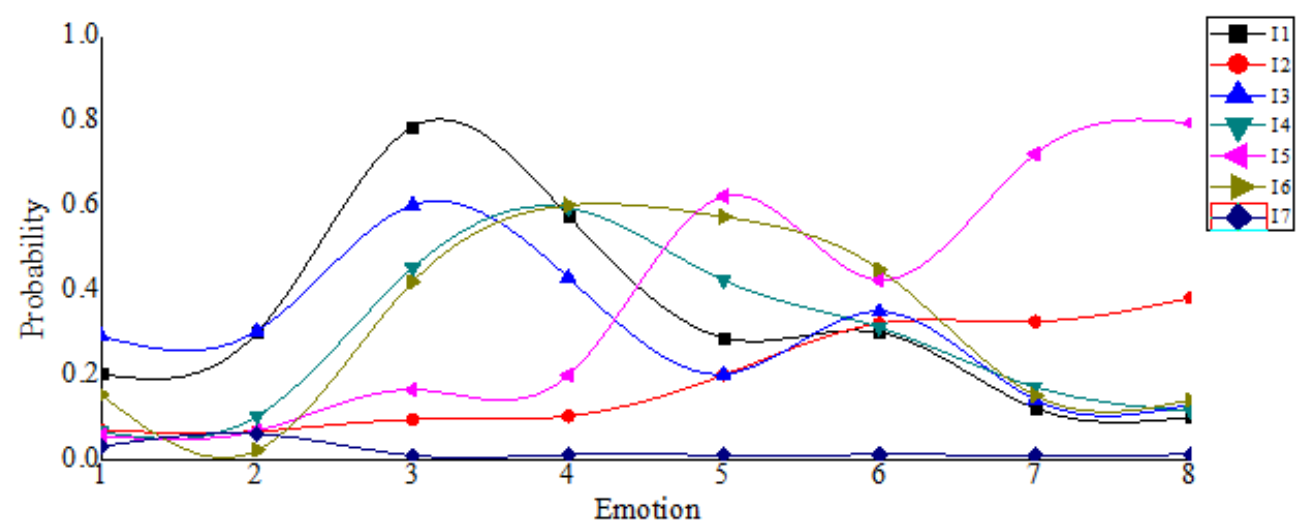

Figure 12. Probability of driver intentions in different emotional states in vehicle group T1.

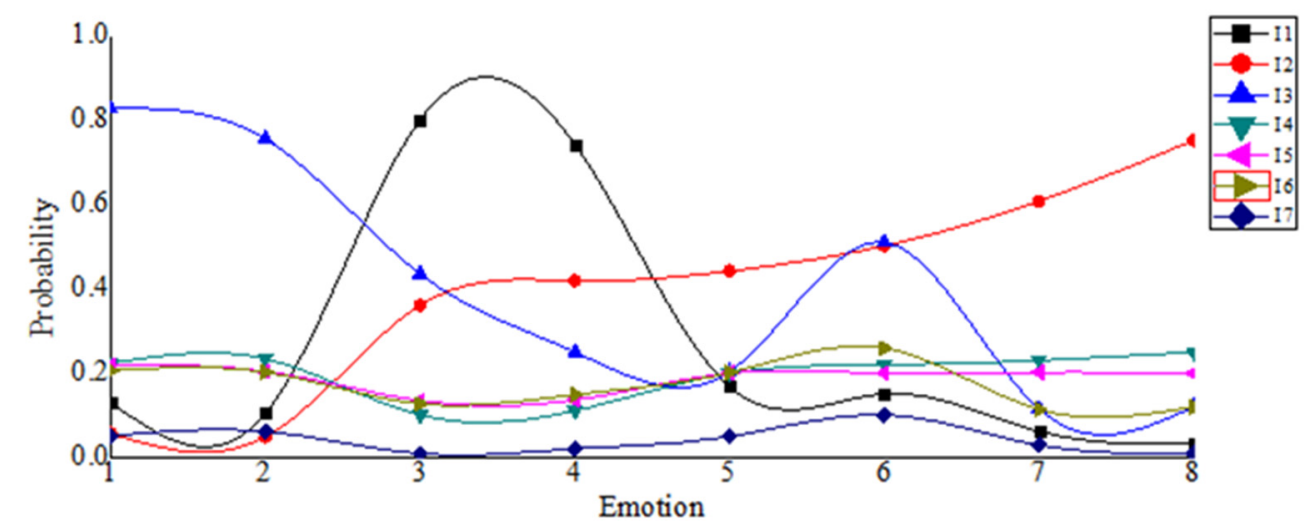

Figure 13. Probability of driver intentions in different emotional states in vehicle group T3.

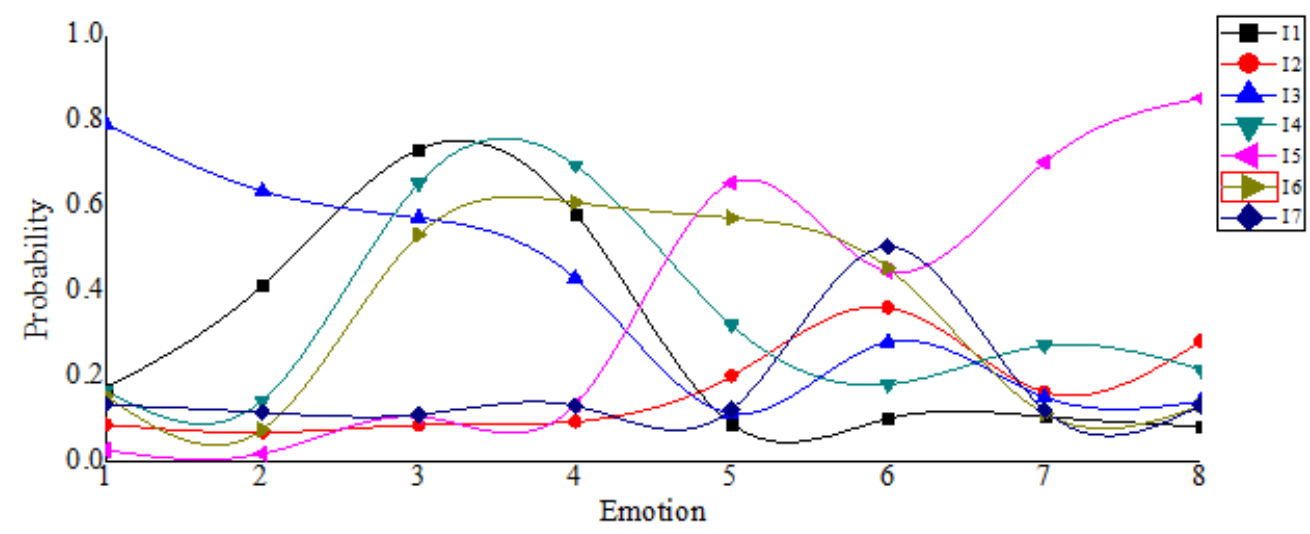

Figure 14. Probability of driver intentions in different emotional states in vehicle group T5.

It was found that for the same vehicle group, the probabilities of driver intention evolution in different emotional states are different. For different vehicle groups, the probabilities of driver intention evolution in the same emotion are also significantly different.

- In the state of fear, drivers tend to be more conservative in their behavioral decisionmaking. Drivers commonly travel at a slow speed and keep a large distance from surrounding vehicles. For example, the intention probability of going-straight with deceleration is 0.828 in the vehicle group T3. This suggests that although the traffic volume is low and the risk is low, drivers still choose to move forward at a low speed. 
- In the state of helplessness, drivers are more likely to suffer from loneliness and lack of dependence. Drivers tend to move forward at a low speed and keep a large distance from surrounding vehicles to ensure safe driving. For example, in the vehicle group $\mathrm{T} 5$, the intention probability of going straight with deceleration is 0.468 . This implies that drivers attempt to keep a safe distance from the vehicle in front by frequently decelerating.

- In the states of relief and pleasure, drivers experience low mood fluctuation. Drivers could remain stable and adhere to rational decision making. For example, in the vehicle group T1, the intention probabilities of going straight with a constant speed are 0.786 and 0.576 in relief and pleasure states, respectively.

- In the state of surprise, drivers have a strong desire to explore the unknown. Therefore, drivers are apt to involve high-frequency acceleration, deceleration, and lane changing in their driving. For example, in the vehicle group T5, the intention probabilities of lane changing with acceleration and lane changing with deceleration are 0.654 and 0.572 , respectively.

- In the state of anxiety, drivers experience inner emotional conflicts which cause muscle tension and restlessness. Therefore, during driving, drivers are prone to accelerate or decelerate frequently to change the surrounding traffic situation. For example, in the vehicle group T5, the intention probabilities of lane changing with acceleration and lane changing with deceleration are 0.446 and 0.455 , respectively.

- In the state of contempt, drivers are more likely to feel superior and condescending to others. Therefore, drivers tend to seek high-speed excitement and make frequent lane changes, even in dangerous traffic situations. For example, in the vehicle group T1, the intention probability of lane changing with acceleration is 0.722 .

- In the state of anger, drivers usually become involved in high-speed traveling, and keep a small following distance. Drivers are more likely to present aggressive behaviors, such as changing lanes with acceleration. For example, in the vehicle group T5, the intention probability of lane changing with acceleration is 0.853 .

\section{Conclusions}

This study identified driver intention emergence characteristics by analyzing driver intention probabilities in different emotions, based on the multi-source data of the humanvehicle-environment dynamic on a two-lane road. The generation mechanism of a driver's intention was analyzed considering the driver's mental state and driving environment. Moreover, a driver intention emergence model with emotion was developed using a threelayer feedforward neural network. The results showed that for the same vehicle group, the probabilities of driver intentions in different emotional states are significantly different. For different vehicle groups, the probabilities of driver intentions in the same emotion are also significantly different.

The findings of this study can be used to improve drivers' behaviors in order to encourage more efficient, safe driving and fuel savings. It can also be used to develop intelligent human-machine interactions in vehicles. Specifically, this would be practically realized by an advanced driver-assist system using the developed model in this study to correctly predict drivers' behavioral intentions. The system would consist of a set of equipment with LIDAR, ultrasonic, cameras, global navigation satellite system, and noncontact physiological sensors. It could collect and process the real-time driver-vehicle-environment data, including drivers' physiological signals, emotional states, driving manipulation, and vehicle group. Further studies are required to increase the effectiveness of the driver intention emergence model through considering more factors such as drivers' temperament, occupation, health status, and other factors related to driving.

Author Contributions: Conceptualization, X.W. and Y.G.; Data curation, S.L.; Formal analysis, X.W., Y.G. and S.L.; Funding acquisition, X.W., Y.G. and Q.Y.; Investigation, Y.G. and S.L.; Methodology, X.W., Y.G. and C.B.; Project administration, X.W.; Resources, X.W. and S.L.; Supervision, X.W.; Validation, C.B., Q.Y. and X.B.; Visualization, S.L.; Writing-original draft, Y.G. and S.L.; Writing- 
review and editing, Y.G. and X.B. All authors have read and agreed to the published version of the manuscript.

Funding: This research was funded by the Joint Laboratory for Internet of Vehicles, Ministry of Education-China Mobile Communications Corporation (grant number ICV-KF2018-03), the Qingdao Top Talent Program of Entrepreneurship and Innovation (grant number 19-3-2-8-zhc), the National Natural Science Foundation of China (grant numbers 71901134, 61074140, 52072214 ), and the Natural Key R\&D Program of China (grant number 2017YFC0803802).

Institutional Review Board Statement: The study was conducted according to the guidelines of the Declaration of Helsinki, and approved by the Institutional Review Board of the College of Electromechanical Engineering at Qingdao University of Science and Technology. (Date of approval was April 25, 2019).

Informed Consent Statement: Informed consent was obtained from all subjects involved in the study.

Data Availability Statement: The data used to support the findings of this study are available from the corresponding author upon request.

Conflicts of Interest: The authors declare that there is no conflict of interest regarding the publication of this paper.

\section{References}

1. Tideman, M.; Voort, V.; Arem, V. A new scenario based approach for designing driver support systems applied to the design of a lane change support system. Transp. Res. Part C Emerg. Technol. 2010, 18, 247-258. [CrossRef]

2. Mohiyeddini, C.; Pauli, R.; Bauer, S. The role of emotion in bridging the intention-behaviour gap: The case of sports participation. Psychol. Sport Exerc. 2009, 10, 226-234. [CrossRef]

3. Chen, H.; Yeh, S.; Huan, T. Nostalgic emotion, experiential value, brand image, and consumption intentions of customers of nostalgic-themed restaurants. J. Bus. Res. 2014, 67, 354-360. [CrossRef]

4. Ladhari, R.; Souiden, N.; Dufour, B. The role of emotions in utilitarian service settings: The effects of emotional satisfaction on product perception and behavioral intentions. J. Retail. Consum. Serv. 2017, 34, 10-18. [CrossRef]

5. Harth, N.; Leach, C.; Kessler, T. Guilt, anger, and pride about in-group environmental behaviour: Different emotions predict distinct intentions. J. Environ. Psychol. 2013, 34, 18-26. [CrossRef]

6. Yang, J.; Zeng, X.; Hu, P. Study on the regulation of festival environment-Based on the perspective of the relationship among consumption emotions, perceived value and behavior intention. Enterp. Econ. 2010, 11, 158-160.

7. Chen, S. The Effects on Matching- Relationship of Behavior Intention and Outcome to Children's Judgment and Attribution of Moral Emotion. Psychol. Explor. 2000, 3, 44-48.

8. Lethaus, F.; Baumann, M.; Lemmer, K. A comparison of selected simple supervised learning algorithms to predict driver intent based on gaze data. Neurocomputing 2013, 121, 108-130. [CrossRef]

9. Helman, S.; Kinnear, N.A.; McKenna, F.P.; Allsop, R.E.; Horswill, M.S. Changes in self-reported driving intentions and attitudes while learning to drive in Great Britain. Accid. Anal. Prev. 2013, 59, 425. [CrossRef] [PubMed]

10. Waddell, L.; Wiener, K. What's driving illegal mobile phone use? Psychosocial influences on drivers' intentions to use hand-held mobile phones. Transp. Res. Part F Traffic Psychol. Behav. 2014, 22, 1-11. [CrossRef]

11. Auzoult, L.; Lheureux, F.; Hardy-Massard, S.; Minary, J.P.; Charlois, C. The perceived effectiveness of road safety interventions: Regulation of drivers' behavioral intentions and self-consciousness. Transp. Res. Part F Psychol. Behav. 2015, 34, 29-40. [CrossRef]

12. Wang, X.; Wang, G.; Zhang, J.; Ban, X. Driver's Behavior and Decision-Making Optimization Model in Mixed Traffic Environment. Adv. Mech. Eng. 2015, 7, 759571. [CrossRef]

13. Bai, Q.; Chen, Y.; Bie, Y.; Yan, H. Estimating permitted left-turn capacities with nonstrict priority crossing maneuvers. J. Transp. Eng. Part A Syst. 2021, 147, 04021003. [CrossRef]

14. Bai, Q.; Gao, Z.; Qu, Z.; Tao, C. Modeling for left-lane line extensions at signalized intersections with permitted left-turning phase. J. Transp. Eng. Part A Syst. 2020, 146, 04020079. [CrossRef]

15. Wang, K. Study on the Coupling Mechanism of Vehicle Cluster Situation and Driver's Propensity. Master's Thesis, Shandong University of Technology, Zibo, China, 2015.

16. Wang, X.; Guo, Y.; Bai, C.; Yuan, Q.; Liu, S.; Han, J. Driver's Intention Identification with the Involvement of Emotional Factors in Two-Lane Roads. IEEE Trans. Intell. Transp. Syst. 2020, 22, 6866-6874. [CrossRef]

17. Wang, G. Study on the Driver's Propensity Adapted to Vehicle Safety Warning System. Master's Thesis, Shandong University of Technology, Zibo, China, 2014.

18. Zhang, M. Study of Car-Following Behavior of Novice Drivers under Different Risk Levels Based on Real Car Simulation Platform. Master's Thesis, Shanghai Jiao Tong University, Shanghai, China, 2014. 
19. Da-Lei, W.; Yi-Qing, L.; Mei-Chun, P.; Wen-Ming, J.; Xian-Feng, W. Small Sample Based Calculation of Annual Vehicle Kilometers Traveled. J. Transp. Syst. Eng. Inf. Technol. 2009, 9, 155-160.

20. Yang, J.; Ma, J. A structure optimization framework for feed-forward neural networks using sparse representation. Knowl.-Based Syst. 2016, 109, 61-70. [CrossRef]

21. Paradarami, T.; Bastian, N.; Wightman, J. A hybrid recommender system using artificial neural networks. Expert Syst. Appl. 2017, 83, 300-313. [CrossRef]

22. Ma, D.; Song, X.; Li, P. Daily traffic flow forecasting through a contextual convolutional recurrent neural network modeling inter-and intra-day traffic patterns. IEEE Trans. Intell. Transp. Syst. 2021, 22, 2627-2636. [CrossRef]

23. Ma, D.; Song, X.B.; Zhu, J.; Ma, W. Input data selection for daily traffic flow forecasting through contextual mining and intra-day pattern recognition. Expert Syst. Appl. 2021, 176, 114902. [CrossRef]

24. Johnston, V.S. Why We Feel, the Science of Human Emotions; Perseus Publishing: New York, NY, USA, 1999.

25. Perez-Gaspar, L.; Caballero-Morales, S.; Trujillo-Romero, F. Multimodal emotion recognition with evolutionary computation for human-robot interaction. Expert Syst. Appl. 2016, 66, 42-61. [CrossRef]

26. Siedlecka, E.; Denson, T.F. Experimental methods for inducing basic emotions: A qualitative review. Emot. Rev. 2019, 11, 87-97. [CrossRef]

27. Platt, J.; Cristianini, N.; Shawe-Taylor, J. Large Margin DAGs for Multiclass Classification. Adv. Neural Inf. Process. Syst. 1999, 12, 547-553. 